Marquette University

e-Publications@Marquette

Biomedical Sciences Faculty Research and

Publications

Biomedical Sciences, Department of

6-1-2012

\title{
Glutathione-Mediated Neuroprotection Against Methylmercury Neurotoxicity in Cortical Culture is Dependent on MRP1
}

Travis Rush

Marquette University

Xiaoqian Liu

Marquette University, xiaoqian.1.liu@marquette.edu

Andrew B. Nowakowski

University of Wisconsin - Milwaukee

David H. Petering

University of Wisconsin - Milwaukee

Doug Lobner

Marquette University, doug.lobner@marquette.edu

Accepted version. NeuroToxicology, Vol. 33, No. 3 (June 2012): 476-481. DOI. (C) 2012 Elsevier Inc. Used with permission. 
Marquette University

e-Publications@Marquette

\section{Biomedical Sciences Faculty Research and Publications/College of Health Sciences}

This paper is NOT THE PUBLISHED VERSION; but the author's final, peer-reviewed manuscript. The published version may be accessed by following the link in th citation below.

NeuroToxicology, Vol. 33, No. 3 (June 2012): 476-481. DOI. This article is C Elsevier and permission has been granted for this version to appear in e-Publications@Marquette. Elsevier does not grant permission for this article to be further copied/distributed or hosted elsewhere without the express permission from Elsevier.

\section{Glutathione-mediated Neuroprotection Against Methylmercury Neurotoxicity in Cortical Culture is Dependent on MRP1}

Travis Rush

Department of Biomedical Sciences, Marquette University, Milwaukee, WI

XiaoQian Liu

Department of Biomedical Sciences, Marquette University, Milwaukee, WI

Andrew B. Nowakowski

Department of Chemistry and Biochemistry, University of Wisconsin-Milwaukee, Milwaukee, WI

David H. Petering

Department of Chemistry and Biochemistry, University of Wisconsin-Milwaukee, Milwaukee, WI

Doug Lobner

Department of Biomedical Sciences, Marquette University, Milwaukee, WI

\section{Abstract}

Methylmercury (MeHg) exposure at high concentrations poses significant neurotoxic threat to humans worldwide. The present study investigated the mechanisms of glutathione-mediated attenuation of $\mathrm{MeHg}$ neurotoxicity in primary cortical culture. $\mathrm{MeHg}(5 \mu \mathrm{M})$ caused depletion of mono- and disulfide glutathione in 
neuronal, glial and mixed cultures. Supplementation with exogenous glutathione, specifically glutathione monoethyl ester (GSHME) protected against the MeHg induced neuronal death. MeHg caused increased reactive oxygen species (ROS) formation measured by dichlorodihydrofluorescein (DCF) fluorescence with an early increase at $30 \mathrm{~min}$ and a late increase at $6 \mathrm{~h}$. This oxidative stress was prevented by the presence of either GSHME or the free radical scavenger, trolox. While trolox was capable of quenching the ROS, it showed no neuroprotection. Exposure to $\mathrm{MeHg}$ at subtoxic concentrations $(3 \mu \mathrm{M})$ caused an increase in system $\mathrm{x}_{c}{ }^{-}$mediated ${ }^{14} \mathrm{C}$-cystine uptake that was blocked by the protein synthesis inhibitor, cycloheximide (CHX). Interestingly, blockade of the early ROS burst prevented the functional upregulation of system $\mathrm{x}_{c}^{-}$. Inhibition of multidrug resistance protein-1 (MRP1) potentiated MeHg neurotoxicity and increased cellular MeHg. Taken together, these data suggest glutathione offers neuroprotection against MeHg toxicity in a manner dependent on MRP1-mediated efflux.

\section{Abbreviations}

CPG carboxyphenylglycine

CGN cerebellar granular neurons

$\mathrm{CHX}$ cycloheximide

DCF dichlorodihydrofluorescein

GSHME glutathione monoethyl ester

ICP-MS inductively-coupled plasma mass spectrometry

LDH lactate dehydrogenase

MeHg methylmercury

MRP1 multi-drug resistance protein 1

NAC $N$-acetyl cysteine

ROS reactive oxygen species

\section{Keywords}

Glutathione, Methylmercury, System $x_{c}^{-}$, MRP1, Neurotoxicity, Neuroprotection

\section{Introduction}

Methylmercury ( $\mathrm{MeHg}$ ) is an ubiquitous neurotoxicant posing significant threat to humans when ingested at high concentrations. Exposure to MeHg has been implicated as a factor in the development of neurodegenerative diseases such as Alzheimer's Disease, Parkinson's Disease, and amyotrophic lateral sclerosis (ALS) (Hock et al., 1998, Monnet-Tschudi et al., 2006, Praline et al., 2007). Humans are primarily exposed to MeHg through consumption of contaminated fish and shellfish. MeHg is formed by bacterial methylation of inorganic mercury in aquatic sediments (Clarkson, 1997). MeHg then travels up the food chain and accumulates in fish and shellfish, and is subsequently consumed by humans (Mahaffey et al., 2004). There is clear evidence for neurological deficits following exposure to high levels of mercury. For example, a large release of mercury into Minamata Bay in Japan led to toxic levels of exposure and severe injury to the local population, including neurological dysfunctions (Harada, 1995, Tsuda et al., 2009). In a separate incident, methylmercury-contaminated seed grain was sent to a population in Iraq with its consumption leading to severe neurological injuries (Myers et al., 2000). Acute exposure to high levels of mercury is known to target cerebellar granular neurons (Fonnum and Lock, 2000), however, due to the location of damage in neurodegenerative diseases, there is also interest in the effects of mercury on cortical neurons. While humans are exposed to other forms of mercury, such as elemental mercury found in dental amalgam restorations, or ethylmercury found in the commercial preservative, thimerosal, $\mathrm{MeHg}$ is of specific concern because it is easily absorbed in the gastrointestinal tract, and readily traverses the blood-brain barrier (BBB) (Bridges and Zalups, 2005, Bridges and Zalups, 2010).

Proposed mechanisms for MeHg neurotoxicity are primarily focused on four possibilities: disruption of intracellular calcium and zinc ion homeostasis, likely involving mitochondrial deficits (Atchison and Hare, 
1994, Kawanai et al., 2009), redox imbalance by increased production of reactive oxygen species and/or by decreasing endogenous cellular antioxidant defenses (Ali et al., 1992, Yee and Choi, 1996, Aschner, 2000, Franco et al., 2007, Amonpatumrat et al., 2008, Wang et al., 2009), direct interactions with free protein sulfhydryl groups (Rooney, 2007), and inhibition of selenoenzymes, especially those involved in redox signaling (Carvalho et al., 2008). The present study is aimed at determining the effects of MeHg on the glutathione (GSH) cycling system and oxidative stress.

Astrocytes are thought to be responsible for de novo synthesis

of glutathione from glutamate, glycineand the rate limiting substrate, cysteine, which is brought into the astrocytes primarily in its oxidized form, cystine (Kranich et al., 1998). Glutathione can be utilized by the cell to reduce reactive oxygen species, for example, superoxide produced as a byproduct of mitochondrial energy production rapidly reacts to form hydrogen peroxide which is then reduced by glutathione to form glutathionedisulfide(GSSG) and water in a reaction catalyzed by glutathione peroxidase. Glutathione may also be utilized as a xenobiotic detoxicant as has been well characterized involving chemotherapeutics in cancertreatment (Salinas and Wong, 1999). That is, glutathione can be directly conjugated to exogenous substrates via a disulfide bond with the free sulfhydryl groups; these reactions are directed by a class of enzymes known as glutathione-Stransferases (GSTs) (Dringen, 2000, Dringen and Hirrlinger, 2003). GSH, GSSG and the glutathione-conjugates are then exported from the cell in a glutathione-dependent manner via multi-drug resistance proteins (MRP), specifically MRP1 in the CNS (Hirrlinger and Dringen, 2005, Minich et al., 2006). These glutathione molecules can then be broken down in the extracellular space by glutathione reductase, amino-peptidase $\mathrm{N}$, or gammaglutamyl transpeptidase. This metabolism produces the substrate cysteine, which can be taken up and utilized by neurons to produce their own glutathione. In this way, neurons are dependent on astrocytes to supply glutathione (Dringen et al., 1999). Since glutathione serves a dual role as both an antioxidant and detoxicant, and both of these roles may offer independent protective mechanisms against $\mathrm{MeHg}$, the present study examines the impact of $\mathrm{MeHg}$ on these aspects of glutathione action.

\section{Materials and methods}

\subsection{Materials}

Timed pregnant Swiss Webster mice were obtained from Charles River Laboratories (Wilmington, DE, USA). Serum was from Atlanta Biologicals (Atlanta, GA, USA). NADPH was from Applichem (Darmstadt, Germany). Radiolabeled ${ }^{14} \mathrm{C}$-cystine was purchased from PerkinElmer (Boston, MA, USA). S-(4)carboxyphenylglycine (CPG) and MK571 were obtained from Tocris Bioscience (Ellisville, MO, USA). DCF was from Molecular Probes (Eugene, OR, USA). All other chemicals were from Sigma (St. Louis, MO, USA).

\subsection{Cortical cell cultures}

Mixed cortical cell cultures containing glial and neuronal cells were prepared from fetal (15-16 day gestation) mice as previously described (Lobner, 2000). Dissociated cortical cells were plated on 24-well plates $\left(2.0 \mathrm{~cm}^{2}\right.$ surface area per well) coated with poly-d-lysine and laminin in Eagles' Minimal Essential Medium (MEM, Earle's salts, supplied glutamine-free) supplemented with $5 \%(\mathrm{v} / \mathrm{v})$ heat-inactivated horse serum, $5 \%$ $(\mathrm{v} / \mathrm{v})$ fetal bovine serum, $2 \mathrm{mM}$ glutamine and d-glucose (total $21 \mathrm{mM}$ ). Neuronal cultures were prepared exactly as above with the addition of $10 \mu \mathrm{M}$ cytosine aribinocide $48 \mathrm{~h}$ after plating to inhibit glial replication (Dugan et al., 1995, Rush et al., 2010). Cultures were plated at approximately 8 hemispheres per plate in a volume of $500 \mu \mathrm{l}$ per well. Glial cultures were prepared as described for mixed cultures from cortical tissue taken from post-natal day 1-3 mice and plated at approximately 3 hemispheres per plate (Choi et al., 1987, Schwartz and Wilson, 1992, Rush et al., 2009). Cultures were maintained in humidified $5 \% \mathrm{CO}_{2}$ incubators at $37^{\circ} \mathrm{C}$. Mice were handled in accordance with a protocol approved by our institutional animal care committee and in compliance with the Public Health Service Policy on Humane Care and Use of Laboratory Animals. All experiments were performed in media identical to growth media except lacking serum (MS) at a volume of $400 \mu \mathrm{l}$ per well. All experiments were performed on mixed cortical cultures, except where noted. 


\subsection{Assay of neuronal death}

Cell death was assessed in cultures by the measurement of lactate dehydrogenase (LDH), released from damaged or destroyed cells, in the extracellular fluid $24 \mathrm{~h}$ after the beginning of the insult. Insults were performed by washing cultures into MS or MS containing the indicated chemicals and incubating overnight. Control LDH levels were subtracted from insult LDH values, and results normalized to $100 \%$ neuronal death caused by $500 \mu \mathrm{M}$ NMDA. Control experiments have shown previously that the efflux of LDH occurring from either necrotic or apoptotic cells is proportional to the number of cells damaged or destroyed (Koh and Choi, 1987, Lobner, 2000). Trypan blue staining indicated that the cell death observed was neuron selective. Cell-free experiments were performed to exclude the possibility of direct inhibition of LDH by MeHg. That is, $\mathrm{MeHg}$ at concentrations up to $10 \mu \mathrm{M}$ do not alter the quantification of an LDH standard.

\subsection{Glutathione assay}

Total cellular glutathione and media glutathione were assayed using a modified enzymatic

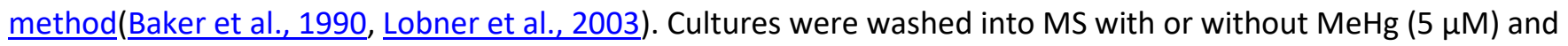
incubated for $6 \mathrm{~h}$. Media samples of $25 \mu \mathrm{l}$ were taken after the drug application period and assayed as aliquots of supernatant below. Cultures were washed with cold $\left(4^{\circ} \mathrm{C}\right) \underline{\text { HEPES }}$ buffered salinesolution, dissolved in $200 \mu \mathrm{l}$ of $1 \%$ sulfosalicylic acid, and centrifuged. A $25 \mu \mathrm{l}$ aliquot of the supernatant was combined with $150 \mu \mathrm{l}$ of $0.1 \mathrm{M}$ phosphate $/ 5 \mathrm{mM}$ EDTA buffer, $10 \mu \mathrm{l}$ of $20 \mathrm{mM}$ dithiobis-2-nitrobenzoic acid, $100 \mu \mathrm{l}$ of $5 \mathrm{mM}$ nicotinamide adenine dinucleotide phosphate $(\mathrm{NADPH})$, and $0.2 \mathrm{U}$ of glutathione reductase. Total glutathione was determined by kinetic analysis of absorbancechanges at $402 \mathrm{~nm}$ for $1.5 \mathrm{~min}$, with concentrations determined by comparison to a standard curve. GSSG was measured as above except samples were treated with 2-vinylpyridine and triethanolaminefor $1 \mathrm{~h}$ prior to beginning the reaction. Cell-free experiments were performed to exclude the possibility of assay inhibition by the experimental reagents used. Due to inter-plate culture variance in absolute glutathione measures data in Table 1 are reported as percent respective intra-plate control glutathione measures. Untreated control values across all culture types were typically in the following ranges (represented as mean nmol/well): 1.99-3.33 cellular GSH, 0.24-0.50 media GSH, 0.14-0.74 cellular GSSG, 0.060-0.16 media GSSG.

Table 1. Effect of MeHg (5 $\mu \mathrm{M})$ on glutathione levels in glial, neuronal and mixed cortical cultures after $6 \mathrm{~h}$ exposure.

\begin{tabular}{|l|l|l|l|l|}
\hline Culture type & GSH & & GSSG & \\
& Cellular & Media & Cellular & Media \\
\hline Mixed & $83.4 \pm 4.7$ & $22.8 \pm 1.0$ & $60.7 \pm 5.7$ & $12.6 \pm 2.4$ \\
\hline Glia & $44.8 \pm 5.2$ & $62.3 \pm 9.8$ & $18.4 \pm 2.9$ & $29.3 \pm 3.7$ \\
\hline Neurons & $59.6 \pm 6.5$ & $36.9 \pm 1.8$ & $20.6 \pm 6.2$ & N/D \\
\hline
\end{tabular}

Results are expressed as $\%$ untreated control (mean $\pm \underline{\mathrm{SEM}}, n=8-16$ ). All values are significantly different from control. N/D = not detectable.

\subsection{Assay for oxidative stress}

Oxidative stress was measured with 5-(and -6)-2'7'-dichlorodihydrofluorescein diacetate (DCF-DA) using a fluorescent plate reader following a modification of a previous method (Wang and Joseph, 1999, Lobner et al., 2007). $10 \mu \mathrm{M}$ of the non-fluorescent, lipophilic compound DCF-DA is added to the cultures for 30 min where it is de-esterified and may be oxidized to fluorescent DCF $\left(2^{\prime}, 7^{\prime}\right.$-dichlorofluorescein). Fluorescence is read using a Fluoroskan Ascent plate reader (Thermo LabSystems) with excitation and emission filters set to $485 \mathrm{~nm}$ and $538 \mathrm{~nm}$, respectively. Background fluorescence (no DCF added) was subtracted and the results normalized to control conditions.

\section{6. ${ }^{14} \mathrm{C}$-cystine uptake}

Radiolabeled cystine uptake was performed as previously described with modifications (Liu et al., 2009). Cultures were exposed to MS containing the indicated drug treatments for $24 \mathrm{~h}$, or for $1 \mathrm{~h}$ followed by $23 \mathrm{~h}$ in 
drug-free MS. $24 \mathrm{~h}$ after the start of the experiment, cultures were washed into HEPES buffered saline solution and immediately exposed to ${ }^{14} \mathrm{C}$-cystine $\left(0.025 \mu \mathrm{Ci} / \mathrm{mL}, 200 \mathrm{nM}\right.$ total cystine) for $20 \mathrm{~min}$. Following ${ }^{14} \mathrm{C}$-cystine exposure, cultures were washed with ice cold HEPES buffered saline solution and dissolved in $250 \mu \mathrm{l}$ warm sodium dodecyl sulfate $(0.1 \%)$. An aliquot $(200 \mu \mathrm{l})$ was removed and added to scintillation fluid for counting. Values were normalized to ${ }^{14} \mathrm{C}$-cystine uptake in untreated controls of the same experimental plate.

\subsection{Determination of MeHg content in cells by ICP-MS}

Following $6 \mathrm{~h}$ exposure to MS with or without the indicated treatments, cultures were washed $3 \times$ with cold MS and then dissolved in $100 \mu \mathrm{l}$ 1\% sulfosalicylic acid. Samples were combined with $900 \mu \mathrm{l} \% \mathrm{HNO}$ containing $500 \mathrm{ppb}$ Au and digested at $70{ }^{\circ} \mathrm{C}$ for $2 \mathrm{~h}$. Digestions were then centrifuged at $6000 \times g$ for 5 min and the supernatants were diluted with an additional $1 \mathrm{~mL}$ of $5 \% \mathrm{HNO}_{3}$. Mercurycontent was determined using a Micromass Platform ICP-MS controlled by MassLynx software (Waters Corporation, Milford, MA). Isotopes ${ }^{198} \mathrm{Hg},{ }^{199} \mathrm{Hg},{ }^{200} \mathrm{Hg},{ }^{201} \mathrm{Hg},{ }^{202} \mathrm{Hg}$, and ${ }^{204} \mathrm{Hg}$ were recorded and total mercury was quantified by comparing sample responses to those produced by commercial standards treated identically to the samples.

\subsection{Statistical analysis}

Differences between test groups were examined for statistical significance by means of one-way ANOVA followed by the Student-Neuman Keuls post hoc analysis, with $p<0.05$ being considered significant.

\section{Results}

Methylmercury $(0.3-10 \mu \mathrm{M})$ exposure induced concentration-dependent neurotoxicity in mixed neuronal and glial cortical cultures $24 \mathrm{~h}$ after onset of insult (Fig. 1). Cell death was assayed by release of the cytosolic enzyme $\underline{L D H} 24 \mathrm{~h}$ after the beginning of the insult. Further, trypan blue staining of these cultures demonstrated that the MeHg-induced toxicity at the concentrations tested was purely neuronal (data not shown). From these data we chose $5 \mu \mathrm{M} \mathrm{MeHg}$ as a moderate insult for subsequent experiments as it allows bidirectional alterations to MeHg-induced neurotoxicity (i.e. neuroprotectionor potentiation). For other experiments we used $3 \mu \mathrm{M}$ MeHg as a low-level insult as no significant neurotoxicity was observed at this concentration (Fig. 1).

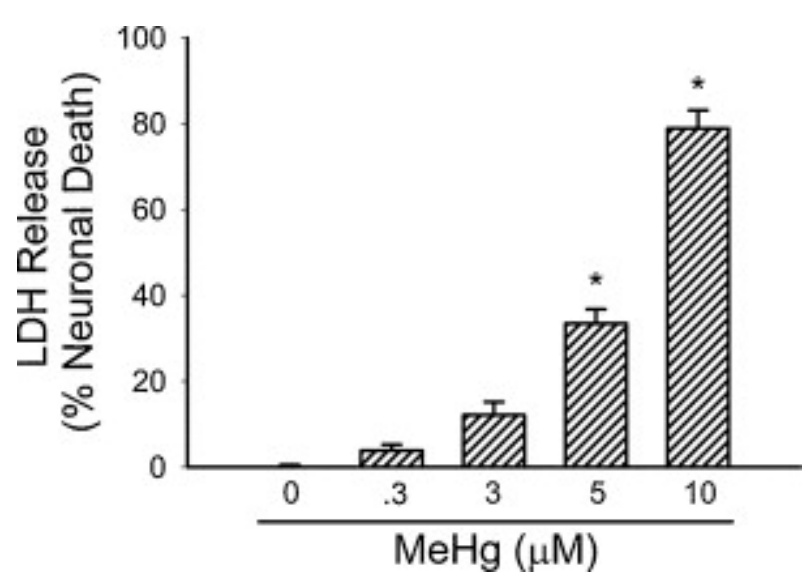

Fig. 1. Concentration response curve of $\mathrm{MeHg}$ (24 $\mathrm{h}$ exposure) on $\underline{\mathrm{LDH}}$ release in primary cortical culture. Results are expressed as mean $+\underline{\text { SEM }}(n=8-16)$. *Significant difference from untreated control.

MeHg toxicity has been shown to cause glutathione depletion in several cell types (Kitahara et al., 1993, Kaur et al., 2006, Amonpatumrat et al., 2008). We found that exposure of mixed neural and glial, pure neuronal and pure glial cultures to $5 \mu \mathrm{M} \mathrm{MeHg}$ significantly decreased total GSH and disulfide glutathione (GSSG) levels in the cells as well as in the extracellular media (Table 1). This glutathionedepletion occurred following a $6 \mathrm{~h}$ MeHg exposure, a time that precedes cell death as no $\underline{\mathrm{LDH}}$ activity was detectable at this time (data not shown). We next tested whether glutathione supplementation or the addition of the free radical scavenger, trolox could protect the neurons. $24 \mathrm{~h}$ co-treatment of $\mathrm{MeHg}$ with glutathione 
monoethyl ester (GSHME) but not trolox, was neuroprotective (Fig. 2). Since glutathione supplementation was protective, and this effect could be due to its nature as an antioxidant or a xenobiotic detoxicant, we next assessed the abilities of GSHME and Trolox to block MeHg-induced oxidative stress.

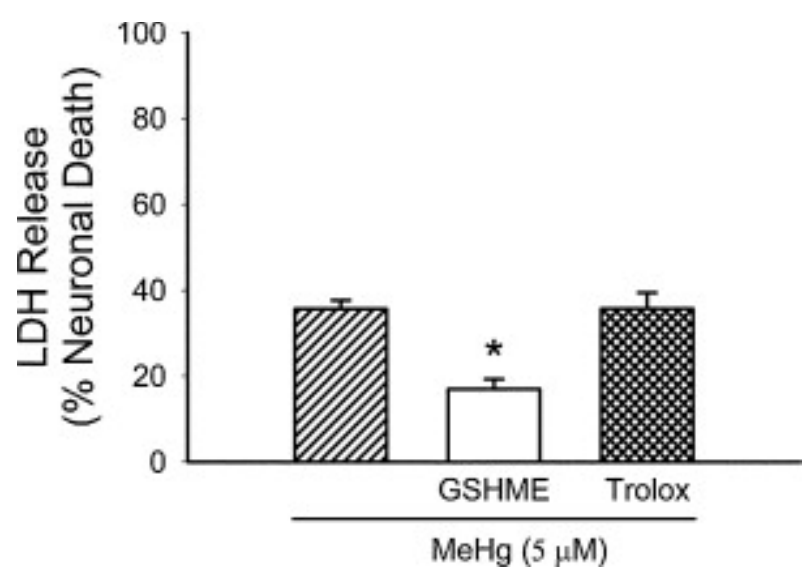

Fig. 2. MeHg-induced neuronal death is attenuated by glutathione monoethyl ester (GSHME, $100 \mu \mathrm{M}$ ) but not trolox $(100 \mu \mathrm{M})$. Results are expressed as mean + $\underline{\mathrm{SEM}}(n=16-20)$. *Significantly different from MeHgalone.

Mixed neuronal and glial cortical cultures exhibited a biphasic pattern of oxidative stress induced by exposure to $5 \mu \mathrm{M}$ MeHg for $0,30,60,180$ or $360 \mathrm{~min}$ (Fig. 3) as measured by fluorescence of DCF.

DCF fluorescence peaked at $30 \mathrm{~min}$, returned to control levels at $3 \mathrm{~h}$ and increased once again by $6 \mathrm{~h}$ following onset of MeHg insult. Both $100 \mu \mathrm{M}$ GSHME and $100 \mu \mathrm{M}$ trolox were capable of preventing the MeHg-induced rise in DCF fluorescence indicative of their ability to block MeHg-induced oxidative stress. However, trolox was much more effective than GSHME actually decreasing the DCF signal well below control levels.

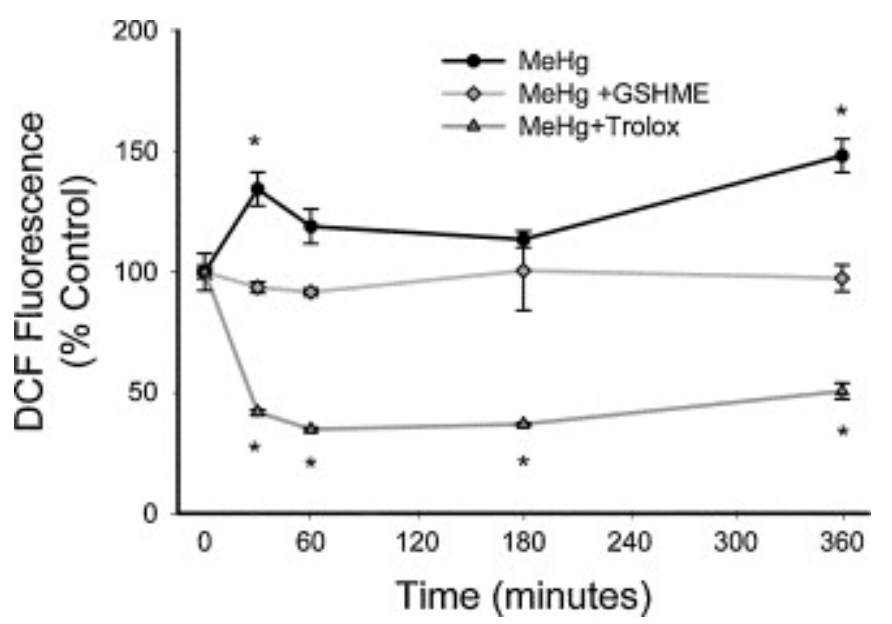

Fig. 3. Timecourse of MeHg ( $5 \mu \mathrm{M})$ induced ROS formation as detected by DCF fluorescence. Results are expressed as mean $+\underline{\text { SEM }(~} n=8-16)$ after normalizing to average fluorescence of untreated controls (i.e. DCF only). *Significantly different from untreated controls.

To further examine MeHg induced alterations to glutathione-related systems, we next tested whether low-level MeHg exposure had an effect on cellular uptake of cystine, the rate limiting substrate for GSH synthesis. Acute exposure to $\mathrm{MeHg}$ had no effect on ${ }^{14} \mathrm{C}$-cystine uptake $(3 \mu \mathrm{M} \mathrm{MeHg}, 20$ min exposure, data not shown); however, 24-h exposure to low-level MeHg (3 $\mu \mathrm{M})$ caused a robust increase in subsequent ${ }^{14} \mathrm{C}$-cystine uptake that was blocked by co-treatment with the protein synthesisinhibitor, cycloheximide (CHX, $500 \mathrm{ng} / \mathrm{mL}$ ) (Fig. 4A). To determine whether the MeHg-induced increase in cystine uptake was mediated by cystine/glutamate exchange, we tested the effects of an inhibitor of this system, CPG ( $300 \mu \mathrm{M}$, present only 
during the 20 min uptake period). The finding that CPG blocked the MeHg-induced increase in cystine uptake indicates that the increase was entirely mediated by functional upregulation of system $\mathrm{x}_{c}^{-}$. When attempting to determine the effect of trolox on the MeHg-induced increase, we found that overnight exposure to trolox alone induced a large increase in subsequent cystine uptake (data not shown). To avoid this complication we used an altered protocol shortening the exposure time to the trolox and/or MeHg to $1 \mathrm{~h}$, followed by a $23 \mathrm{~h}$ incubationin identical exposure media except lacking the trolox and MeHg. 60 min exposure to $\mathrm{MeHg}(3 \mu \mathrm{M})$ produced a similar increase in xCT-mediated cystine uptake $23 \mathrm{~h}$ later (Fig. 4B). Co-treatment with trolox during this period blocked this increase while trolox alone for $60 \mathrm{~min}$ had no effect on uptake.

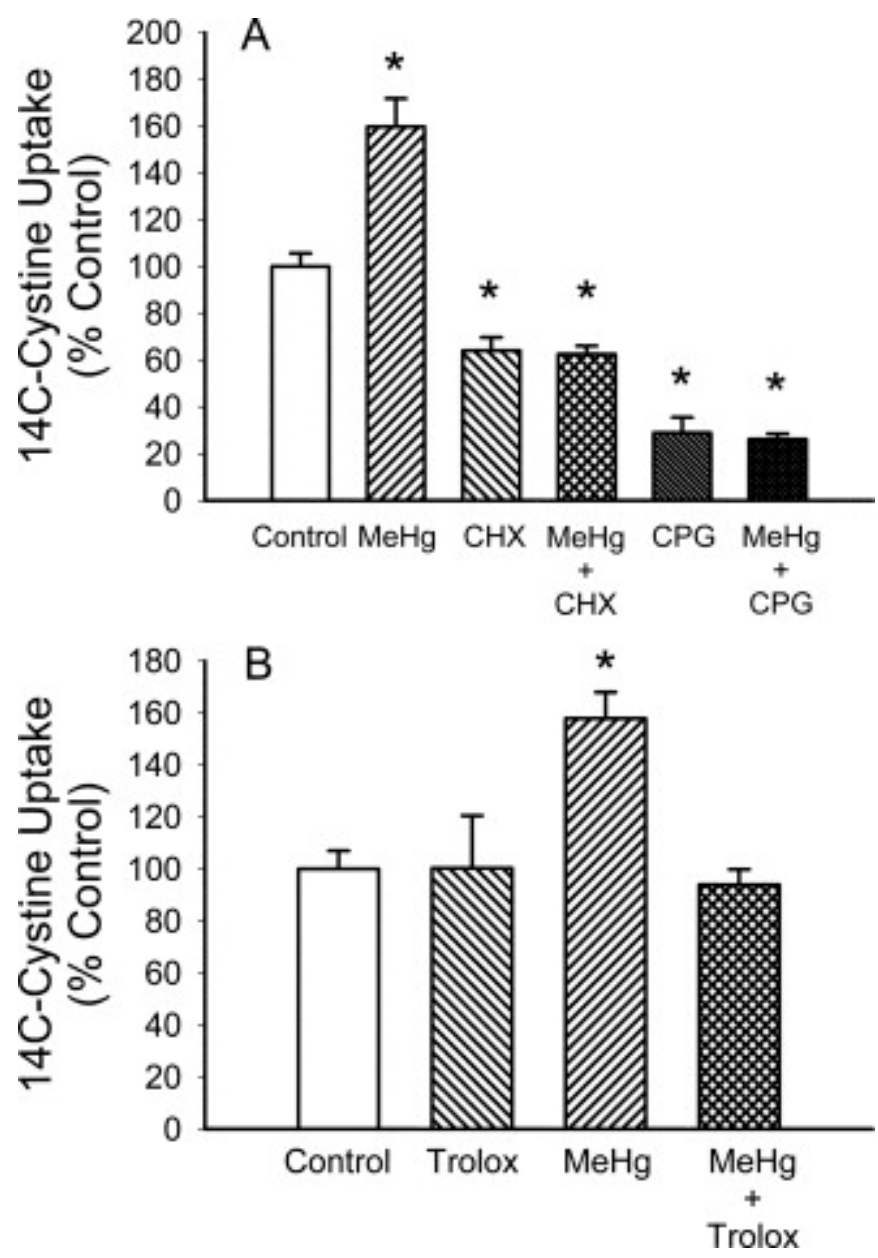

Fig. 4. Subtoxic MeHg exposure causes a robust increase in system $x_{c}{ }^{-}$mediated ${ }^{14} \mathrm{C}$-cystine uptake. (A) Cultures were exposed to $\mathrm{MeHg}(3 \mu \mathrm{M})$, cycloheximide $(\mathrm{CHX}, 500 \mathrm{ng} / \mathrm{mL})$, or in combination for $24 \mathrm{~h}$ prior to measuring uptake. Carboxyphenylglycine (CPG, $300 \mu \mathrm{M})$ was present only during the 20 min uptake period. (B) Cultures were exposed to $\mathrm{MeHg}(3 \mu \mathrm{M})$, trolox $(100 \mu \mathrm{M})$ or in combination for $60 \mathrm{~min}$, washed into drug-free media and incubated overnight $(23 \mathrm{~h})$ prior to assay for cystine uptake. Results are expressed as mean $+\underline{\text { SEM }}(n=8-16)$ after normalizing to untreated control uptake. *Significantly different from control.

It is possible that glutathione conjugates to $\mathrm{MeHg}$ intracellularly and that this conjugate is exported via MRP1. To test whether the export of glutathione is necessary for its protective capabilities, we co-treated cultures with MeHg and an inhibitor of MRP1, MK571. MK571 alone caused no significant cell toxicity; however, by inhibiting MRP1-mediated glutathione export MeHg neurotoxicity was severely potentiated (Fig. 5A).

Supporting the idea that MeHg conjugates to GSH, we found that inhibiting MRP1-mediated glutathione export with MK571 led to a doubling of MeHg accumulation within the cellular compartment (Fig. $5 \mathrm{~B}$ ).

Supplementation with GSHME during the MeHg treatment had no effect on mercury accumulation in the cells (data not shown). 

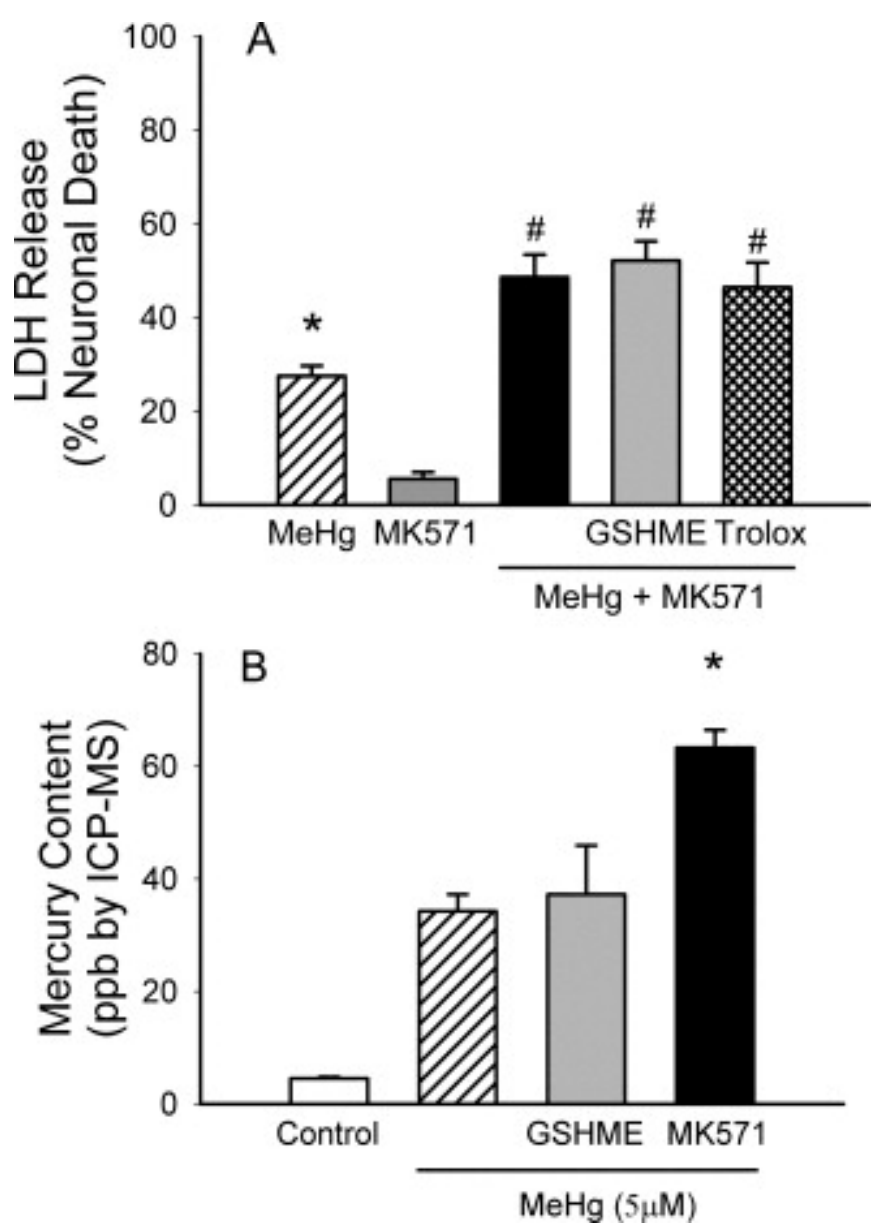

Fig. 5. Blockade of glutathione efflux augments MeHg toxicity and cellular accumulation of mercury. (A) Cultures were exposed to MeHg $(5 \mu \mathrm{M})$, MK571 $(10 \mu \mathrm{M})$, or both in combination with or without GSHME $(100 \mu \mathrm{M})$ for $24 \mathrm{~h}$ at which time samples were taken and neuronal death quantified by $\underline{\mathrm{LDH}}$ release. Results are expressed as mean $+\underline{\text { SEM }}(n=8-12)$. *Significantly different from untreated control, \#significantly different from MeHg-only treated. (B) Cultures were exposed to MeHg $(5 \mu \mathrm{M})$ in the presence of GSHME (100 $\mu \mathrm{M})$ or MK571 (50 $\mu \mathrm{M})$ for $6 \mathrm{~h}$. Samples were then taken and subsequently analyzed for mercury content by ICP-MS. Results are expressed as mean $+\operatorname{SEM}(n=3-6)$.

\section{Discussion}

The present study offers several insights into the contributions of alterations in glutathione cycling and oxidative stress to methylmercury-induced neurotoxicity. We observed significant toxicity with overnight exposure of mixed cortical cultures to $5 \mu \mathrm{M} \mathrm{MeHg}$. This concentration is similar to those used in other studies demonstrating cytotoxicity, oxidative stress and mitochondrial deficits in cortical and cerebellar granular neuron (CGN) cultures (Gasso et al., 2001, Morken et al., 2005, Kaur et al., 2006, Yin et al., 2007). However, it is important to note that CGNs have been shown to be more sensitive to MeHg toxicity (Sarafian and Verity, 1991, Marty and Atchison, 1997, Sakaue et al., 2005). Though not the only factor recognized, the increased sensitivity of CGNs has been posited as attributable to the relatively low glutathione content of these cells (Yee and Choi, 1996, Shafer et al., 2002, Kaur et al., 2007, Wang et al., 2009).

Previous studies have shown that MeHg causes a depletion of cellular glutathione (Yee and Choi, 1996, Franco et al., 2007, Amonpatumrat et al., 2008, Wang et al., 2009). Here, we also report that MeHg depletes both total and disulfide glutathione in cortical culture; total GSH and disulfide GSSG were decreased in cellular and media samples from glia-enriched, neuron-enriched and mixed cultures. GSH can reduce free

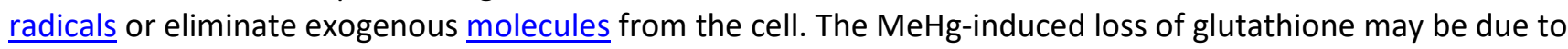
the formation of ROS and subsequent use of GSH by the cells to reduce the oxidative damage. However, since 
reduction of oxidative stress by GSH results in the formation of GSSG, the observed depletion of both GSH and GSSG suggests that the loss of glutathione was not due to oxidative stress. This data is consistent with the hypothesis that glutathione is acting to detoxify MeHg by direct conjugation which may render the utilized GSH molecules undetectable by our assay, and perhaps unrecoverable by the cell.

It has been shown that modulation of cellular thiols (including GSH) alters sensitivity to MeHg in culture (Kaur et al., 2006) and that exogenous supply of glutathione precursors such as $\mathrm{N}$-acetyl-cysteine (NAC) or cystine is neuroprotective (Fujiyama et al., 1994, Kaur et al., 2006, Kaur et al., 2007). Specifically, NAC is able to boost cellular GSH availability and leads to decreased MeHg-induced cytotoxicity and ROS formation. Inhibition of glutathione synthesis with buthionine sulfoxamine (BSO) increases sensitivity to MeHg and increases subsequent ROS formation (Toyama et al., 2011). These studies also demonstrate that modulation of glutathione availability alters cellular ROS formation in response to MeHg insult. Further, this ROS formation is sensitive to antioxidants (Gasso et al., 2001, Shanker and Aschner, 2003, Kaur et al., 2010). Importantly, however, in our hands troloxdoes not offer the neuroprotective capacity that glutathione supplementation with GSHME provides. This suggests that oxidative stress alone is not primary to the toxicity of MeHg under these conditions. Others have shown that trolox does offer neuroprotection against early MeHg-induced mitochondrial deficits detected by MTT metabolism after short, $1 \mathrm{~h}$ exposure (Kaur et al., 2010). Notably, however, these studies did not investigate later timepoints as seen in the present data.

An important action of $\mathrm{MeHg}$ is the inhibition of selenoproteins. Among the selenoproteins that $\mathrm{MeHg}$ has been shown to inhibit are thioredoxin reductase and thioredoxin-glutathione peroxidase(Carvalho et al., 2008, Reeves and Hoffmann, 2009, Branco et al., 2011). In fact, the IC50 for the inhibition of thioredoxin reductase 1 is $9 \mathrm{nM}$ (Carvalho et al., 2008); therefore, at the concentrations of MeHg used in the current study this enzyme, and similar selenoproteins, are likely greatly inhibited. Therefore, it is clear that interaction with selenoproteins could be a mechanism of MeHg induced GSH depletion and oxidative stress. However, while preincubation with selenomethionine has been shown to attenuate $\mathrm{MeHg}$ induced production of reactive oxygen species, it did not prevent depletion of GSH (Kaur et al., 2009). Although, dietary selenium is known to be protective against MeHg exposure (Ralston and Raymond, 2010).

MeHg can also inhibit other enzymes important for the cells response to oxidative stress, such as thioredoxin and glutathione reductase (Carvalho et al., 2008). However, the inhibition of glutathione reductase was found to only occur in vitro and not in cells treated with MeHg (Carvalho et al., 2008).

We also examined the function of the cystine-glutamate antiporter in response to MeHg exposure by radiolabeled ${ }^{14} \mathrm{C}$-cystine uptake. As discussed above, cystine uptake is an important aspect of glutathione synthesis and subsequent cycling. Low-level $\mathrm{MeHg}$ insult induced a robust increase in cystine uptake. This functional upregulation of $\mathrm{XCT}$ was blocked by $\mathrm{CHX}$ suggesting that the upregulation requires translation of new protein. As well, CPG blocked this increased radioliganduptake to CPG-treated control levels suggesting the entire MeHg-induced increase was mediated by system $\mathrm{x}_{c}{ }^{-}$. Trolox also blocked the MeHg-increased cystine uptake. Considering that upregulation of $\mathrm{xCT}$ is likely an endogenous mechanism of cytoprotection in response to MeHg-insult, the fact that trolox blocks upregulation of XCT may account for the inability of trolox to protect against the neuronal injury. The most likely mechanism for increased system $\mathrm{x}_{c}{ }^{-}$activity is upregulation of $\mathrm{xCT}$ by activation of the nrf2-ARE pathway (Mysona et al., 2009, Wang et al., 2009, Qin et al., 2010). Consistent with this explanation is a recent report demonstrating nrf2-dependent methylmercury detoxificationin hepatocytes (Toyama et al., 2011) and an earlier report in astrocytes (Wang et al., 2009).

The current data indicate that blockade of oxidative stress alone is insufficient to prevent $\mathrm{MeHg}$ induced neuronal death, and that elimination of MeHg from the cell by conjugation to GSH is necessary. Consistent with this, inhibition of glutathione export via the MRP1 inhibitor, MK571, not only potentiated MeHginduced neurotoxicity, but this potentiation was associated with increased accumulation of MeHg in the cell. Similar results have been reported in primary mouse hepatocytes where it was found that upregulation of MRP1 by isothiocyanates decreased MeHg accumulation and toxicity (Toyama et al., 2011). Interestingly, and consistent with a previous report using NAC (Kaur et al., 2006), glutathione supplementation with GSHME did not affect MeHg accumulation in the cell. Trolox has previously been reported to have no effect on MeHg accumulation nor on glutathione availability (Kaur et al., 2010); these and the present data, support the 
assertion that oxidative stress is not primary to MeHg-induced neurotoxicity. Rather, conjugation with MeHg and subsequent export of glutathione from the cytosol is crucial to alleviate or prevent MeHg-induced neurotoxicity and that solely blocking oxidative stress is not likely to offer significant neuroprotection.

\section{Conflict of interest statement}

The authors declare that there are no conflicts of interest.

\section{References}

Ali et al., 1992 S.F. Ali, C.P. LeBel, S.C. Bondy Reactive oxygen species formation as a biomarker of methylmercury and trimethyltin neurotoxicity Neurotoxicology, 13 (1992), pp. 637-648

Amonpatumrat et al., 2008

S. Amonpatumrat, H. Sakurai, P. Wiriyasermkul, N.Khunweeraphong, S. Nagamori, H. Tanaka, et al. I-glutamate enhances methylmercury toxicity by synergistically increasing oxidative stress $J$ Pharmacol Sci, 108 (2008), pp. 280-289

Aschner, 2000 M. Aschner Astrocytic swelling, phospholipase A2, glutathione and glutamate: interactions in methylmercury-induced neurotoxicity Cell Mol Biol (Noisy-le-grand), 46 (2000), pp. 843-854

Atchison and Hare, 1994 W.D. Atchison, M.F. Hare Mechanisms of methylmercury-induced neurotoxicity FASEB J, 8 (1994), pp. 622-629

Baker et al., 1990 M.A. Baker, G.J. Cerniglia, A. Zaman Microtiter plate assay for the measurement of glutathione and glutathione disulfide in large numbers of biological samples Anal Biochem, 190 (1990), pp. 360-365

Branco et al., 2011 V. Branco, J. Canario, J. Lu, A. Holmgren, C. Carvalho Mercury and selenium interaction in vivo: effects on thioredoxin reductase and glutathione peroxidase Free Radic Biol Med (2011) [Epub ahead of print]

Bridges and Zalups, 2005 C.C. Bridges, R.K. Zalups Molecular and ionic mimicry and the transport of toxic metals Toxicol Appl Pharmacol, 204 (2005), pp. 274-308

Bridges and Zalups, 2010 C.C. Bridges, R.K. Zalups Transport of inorganic mercury and methylmercury in target tissues and organs J Toxicol Environ Health B Crit Rev, 13 (2010), pp. 385-410

Carvalho et al., 2008 C.M.L. Carvalho, E.-H. Chew, S.I. Hashemy, J. Lu, A. Holmgren Inhibition of the human thioredoxin system. A molecular mechanism of mercury toxicity J Biol Chem, 283 (2008), pp. 1191311923

Choi et al., 1987 D.W. Choi, M. Maulucci-Gedde, A.R. Kriegstein Glutamate neurotoxicity in cortical cell culture J Neurosci, 7 (1987), pp. 357-368

Clarkson, 1997 T.W. Clarkson The toxicology of mercury Crit Rev Clin Lab Sci, 34 (1997), pp. 369-403

Dringen, 2000 R. Dringen Metabolism and functions of glutathione in brain Prog Neurobiol, 62 (2000), pp. 649671

Dringen and Hirrlinger, 2003 R. Dringen, J. Hirrlinger Glutathione pathways in the brain Biol Chem, 384 (2003), pp. 505-516

Dringen et al., 1999 R. Dringen, B. Pfeiffer, B. Hamprecht Synthesis of the antioxidant glutathione in neurons: supply by astrocytes of CysGly as precursor for neuronal glutathione J Neurosci, 19 (1999), pp. 562-569

Dugan et al., 1995 L.L. Dugan, V.M. Bruno, S.M. Amagasu, R.G. Giffard Glia modulate the response of murine cortical neurons to excitotoxicity: glia exacerbate AMPA neurotoxicity J Neurosci, 15 (1995), pp. 45454555

Fonnum and Lock, 2000 F. Fonnum, E.A. Lock Cerebellum as a target for toxic substances Toxicol Lett, 15 (2000), pp. 9-16

Franco et al., 2007 J.L. Franco, H.C. Braga, J. Stringari, F.C. Missau, T. Posser, B.G. Mendes, et al. Mercurialinduced hydrogen peroxide generation in mouse brain mitochondria: protective effects of quercetin Chem Res Toxicol, 20 (2007), pp. 1919-1926

Fujiyama et al., 1994 J. Fujiyama, K. Hirayama, A. Yasutake Mechanism of methylmercury efflux from cultured astrocytes Biochem Pharmacol, 47 (1994), pp. 1525-1530 
Gasso et al., 2001 S. Gasso, R.M. Cristofol, G. Selema, R. Rosa, E. Rodriguez-Farre, C.Sanfeliu Antioxidant compounds and $\mathrm{Ca}(2+)$ pathway blockers differentially protect against methylmercury and mercuric chloride neurotoxicity J Neurosci Res, 66 (2001), pp. 135-145

Harada, 1995 M. Harada Minamata disease: methylmercury poisoning in Japan caused by environmental pollution Crit Rev Toxicol, 25 (1995), pp. 1-24

Hirrlinger and Dringen, $2005 \mathrm{~J}$. Hirrlinger, R. Dringen Multidrug resistance protein 1-mediated export of glutathione and glutathione disulfide from brain astrocytes Methods Enzymol, 400 (2005), pp. 395-409

Hock et al., 1998 C. Hock, G. Drasch, S. Golombowski, F. Muller-Spahn, B. WillershausenZonnchen, P. Schwarz, et al. Increased blood mercury levels in patients with Alzheimer's disease J Neural Transm, 105 (1998), pp. 59-68

Kaur et al., 2006 P. Kaur, M. Aschner, T. Syversen Glutathione modulation influences methyl mercury induced neurotoxicity in primary cell cultures of neurons and astrocytes Neurotoxicology, 27 (2006), pp. 492500

Kaur et al., 2007 P. Kaur, M. Aschner, T. Syversen Role of glutathione in determining the differential sensitivity between the cortical and cerebellar regions towards mercury-induced oxidative stress Toxicology, 230 (2007), pp. 164-177

Kaur et al., 2009 P. Kaur, L. Evje, M. Aschner, T. Syversen The in vitro effects of selenomethionine on methylmercury-induced neurotoxicity Toxicol In Vitro, 23 (2009), pp. 378-385

Kaur et al., 2010 P. Kaur, L. Evje, M. Aschner, T. Syversen The in vitro effects of Trolox on methylmercuryinduced neurotoxicity Toxicology, 276 (2010), pp. 73-78

Kawanai et al., 2009 T. Kawanai, M. Satoh, K. Murao, Y. Oyama Methylmercury elicits intracellular Zn2+ release in rat thymocytes: its relation to methylmercury-induced decrease in cellular thiol content Toxicol Lett, 191 (2009), pp. 231-235

Kitahara et al., 1993 J. Kitahara, Y. Seko, N. Imura Possible involvement of active oxygen species in selenite toxicity in isolated rat hepatocytes Arch Toxicol, 67 (1993), pp. 497-501

Koh and Choi, 1987 J.Y. Koh, D.W. Choi Quantitative determination of glutamate mediated cortical neuronal injury in cell culture by lactate dehydrogenase efflux assay J Neurosci Methods, 20 (1987), pp. 83-90

Kranich et al., 1998 O. Kranich, R. Dringen, M. Sandberg, B. Hamprecht Utilization of cysteine and cysteine precursors for the synthesis of glutathione in astroglial cultures: preference for cystine Glia, 22 (1998), pp. $11-18$

Liu et al., 2009 X. Liu, T. Rush, J. Zapata, G.D. Lobner Beta-N-methylamino-I-alanine induces oxidative stress and glutamate release through action on system Xc(-) Exp Neurol, 217 (2009), pp. 429-433

Lobner, 2000 D. Lobner Comparison of the LDH and MTT assays for quantifying cell death: validity for neuronal cell death J Neurosci Meth, 96 (2000), pp. 147-152

Lobner et al., 2003 D. Lobner, S. Golner, J. Hjelmhaug Neurotrophic factor effects on oxidative stress-induced neuronal death Neurochem Res, 28 (2003), pp. 749-756

Lobner et al., 2007 D. Lobner, P.M. Piana, A.K. Salous, R.W. Peoples Beta-N-methylamino-I-alanine enhances neurotoxicity through multiple mechanisms Neurobiol Dis, 25 (2007), pp. 360-366

Mahaffey et al., 2004 K.R. Mahaffey, R.P. Clickner, C.C. Bodurow Blood organic mercury and dietary mercury intake: National Health and Nutrition Examination Survey, 1999 and 2000 Environ Health Perspect, 112 (2004), pp. 562-570

Marty and Atchison, 1997 M.S. Marty, W.D. Atchison Pathways mediating Ca2+ entry in rat cerebellar granule cells following in vitro exposure to methyl mercury Toxicol Appl Pharmacol, 147 (1997), pp. 319-330

Minich et al., 2006 T. Minich, J. Riemer, J.B. Schulz, P. Wielinga, J. Wijnholds, R. Dringen The multidrug resistance protein 1 (Mrp1), but not Mrp5, mediates export of glutathione and glutathione disulfide from brain astrocytes $J$ Neurochem, 97 (2006), pp. 373-384

Monnet-Tschudi et al., 2006 F. Monnet-Tschudi, M.G. Zurich, C. Boschat, A. Corbaz, P. Honegger Involvement of environmental mercury and lead in the etiology of neurodegenerative diseases Rev Environ Health, 21 (2006), pp. 105-117 
Morken et al., 2005 T.S. Morken, U. Sonnewald, M. Aschner, T. Syversen Effects of methylmercury on primary brain cells in mono- and co-culture Toxicol Sci, 87 (2005), pp. 169-175

Myers et al., 2000 G.J. Myers, P.W. Davidson, C. Cox, C. Shamlaye, E. Cernichiari, T.W.Clarkson Twenty-seven years studying the human neurotoxicity of methylmercury exposure Environ Res, 83 (2000), pp. 275285

Mysona et al., 2009 B. Mysona, Y. Dun, J. Duplantier, V. Ganapathy, S.B. Smith Effects of hyperglycemia and oxidative stress on the glutamate transporters GLAST and system $\mathbf{x}_{\mathbf{c}}{ }^{-}$in mouse retinal Muller glial cells Cell Tissue Res, 335 (2009), pp. 477-488

Praline et al., 2007 J. Praline, A.M. Guennoc, N. Limousin, H. Hallak, B. de Toffol, P. Corcia ALS and mercury intoxication: a relationship? Clin Neurol Neurosurg, 109 (2007), pp. 880-883

Qin et al., 2010 Z. Qin, E. Freitas, R. Sullivan, S. Mohan, R. Bacelieri, D. Branch, et al. Upregulation of xCT by KSHV-encoded microRNAs facilitates KSHV dissemination and persistence in an environment of oxidative stress PLoS Pathog, 6 (2010), p. e1000742

Ralston and Raymond, 2010 N.V. Ralston, L.J. Raymond Dietary selenium's protective effects against methylmercury toxicity Toxicology, 278 (2010), pp. 112-123

Reeves and Hoffmann, 2009 M.A. Reeves, P.R. Hoffmann The human selenoproteome: recent insights into functions and regulation Cell Mol Life Sci, 66 (2009), pp. 2457-2478

Rooney, 2007 J.P. Rooney The role of thiols, dithiols, nutritional factors and interacting ligands in the toxicology of mercury Toxicology, 234 (2007), pp. 145-156

Rush et al., 2009 T. Rush, J. Hjelmhaug, D. Lobner Effects of chelators on mercury, iron, and lead neurotoxicity in cortical culture Neurotoxicology, 30 (2009), pp. 47-51

Rush et al., 2010 T. Rush, X.Q. Liu, J. Hjelmhaug, D. Lobner Mechanisms of chlorpyrifos and diazinon induced neurotoxicity in cortical culture Neuroscience, 166 (2010), pp. 899-906

Sakaue et al., 2005 M. Sakaue, M. Okazaki, S. Hara Very low levels of methylmercury induce cell death of cultured rat cerebellar neurons via calpain activation Toxicology, 213 (2005), pp. 97-106

Salinas and Wong, 1999 A.E. Salinas, M.G. Wong Glutathione S-transferases - a review Curr Med Chem, 6 (1999), pp. 279-309

Sarafian and Verity, 1991 T. Sarafian, M.A. Verity Oxidative mechanisms underlying methyl mercury neurotoxicity Int J Dev Neurosci, 9 (1991), pp. 147-153

Schwartz and Wilson, 1992 J.P. Schwartz, D.J. Wilson Preparation and characterization of type 1 astrocytes cultured from adult rat cortex, cerebellum, and striatum Glia, 5 (1992), pp. 75-80

Shafer et al., 2002 T.J. Shafer, C.A. Meacham, S. Barone Jr. Effects of prolonged exposure to nanomolar concentrations of methylmercury on voltage-sensitive sodium and calcium currents in PC12 cells Brain Res Dev Brain Res, 136 (2002), pp. 151-164

Shanker and Aschner, 2003 G. Shanker, M. Aschner Methylmercury-induced reactive oxygen species formation in neonatal cerebral astrocytic cultures is attenuated by antioxidants Brain Res Mol Brain Res, 110 (2003), pp. 85-91

Toyama et al., 2011 T. Toyama, Y. Shinkai, A. Yasutake, K. Uchida, M. Yamamoto, Y. Kumagai Isothiocyanates reduce mercury accumulation via an Nrf2-dependent mechanism during exposure of mice to methylmercury Environ Health Perspect (2011)

Tsuda et al., 2009 T. Tsuda, T. Yorifuji, S. Takao, M. Miyai, A. Babazono Minamata disease: catastrophic poisoning due to a failed public health response J Public Health Policy, 30 (2009), pp. 54-67

Wang and Joseph, 1999 H. Wang, J.A. Joseph Quantifying cellular oxidative stress by dichlorofluorescein assay using microplate reader Free Radic Biol Med, 27 (1999), pp. 612-616

Wang et al., 2009 L. Wang, H. Jiang, Z. Yin, M. Aschner, J. Cai Methylmercury toxicity and Nrf2-dependent detoxification in astrocytes Toxicol Sci, 107 (2009), pp. 135-143

Yee and Choi, 1996 S. Yee, B.H. Choi Oxidative stress in neurotoxic effects of methylmercury poisoning Neurotoxicology, 17 (1996), pp. 17-26 
Yin et al., 2007 Z. Yin, D. Milatovic, J.L. Aschner, T. Syversen, J.B. Rocha, D.O. Souza, et al. Methylmercury induces oxidative injury, alterations in permeability and glutamine transport in cultured astrocytes Brain Res, 1131 (2007), pp. 1-10 